\title{
Channel length assisted symbol synchronization for OFDM systems in multipath fading channels
}

\author{
Wen-Long Chin
}

\begin{abstract}
Despite the promising role of orthogonal frequency-division multiplexing (OFDM) technology in communication systems, its synchronization in multipath fading channels remains an important and challenging issue. This work describes a novel synchronization algorithm that exploits channel length information for use in OFDM systems. A timing function that can identify the ISI-free region and subsequently the channel length is also developed based on both the redundancy of the cyclic prefix (CP), and the drastic increase in intersymbol interference (ISI) that rises with symbol timing error in multipath fading channels. Knowledge of the channel length information allows the symbol timing to be safely set in the middle of the ISI-free region, without any ISI. Simulation results indicate that the maximum value of the timing function occurs at the correct timing offset when the signal-to-noise ratio (SNR) is high. From low- to medium SNRs, the correct timing offset is guaranteed when the signal power induced by the channel tap is more significant than the noise power. Furthermore, an efficient search algorithm is derived to reduce the search complexity (search time and computation complexity).
\end{abstract}

Keywords: Channel length, Cyclic prefix (CP), Orthogonal frequency-division multiplexing (OFDM), Symbol synchronization

\section{Introduction}

Orthogonal frequency-division multiplexing (OFDM) is a promising technology for broadband transmission. However, OFDM systems are sensitive to synchronization errors that may destroy the orthogonality among all sub-carriers. Accordingly, intercarrier interference (ICI) and intersymbol interference (ISI) are introduced by synchronization errors [1-4]. First, the uncertain OFDM symbol arrival time introduces a symbol timing offset, which is estimated by the coarse symbol timing offset [5] and fine symbol timing offset [6,7]. Second, the mismatch between the carrier frequencies of the oscillators of the transmitter and the receiver generates a carrier frequency offset (CFO), necessitating the elimination of the resulting fractional CFO [5], integral CFO [8,9], and residual CFO [6,7]. Moreover, the mismatch between the sampling clocks of the digital-to-analog converter (DAC) and the analog-to-digital converter (ADC) introduces a sampling clock frequency offset [7].

Correspondence: johnsonchin@pchome.com.tw

Department of Engineering Science, National Cheng Kung University, No. 1 University Road, Tainan City 701, Taiwan

\section{Springer}

The estimation of symbol timing is essential to the overall OFDM synchronization process, because a poor estimate of symbol timing severely degrades the signalto-interference-and-noise ratio (SINR) [2,7]. Besides ISI, extra ICI is also introduced owing to a loss of orthogonality. The symbol timing is estimated to identify the correct starting position of the OFDM symbol for the fast Fourier transform (FFT) operation. The timing offset is assumed to be an integer and may be set anywhere within an OFDM symbol.

Synchronization algorithms have been extensively reported for OFDM. A good survey can be found in [10]. Some works are briefly described here. Specially designed training preambles in $[6,11]$ can be used for symbol synchronization. Although an accurate estimate can be made, the bandwidth efficiency is reduced by adding a preamble. To eliminate such a reduction, algorithms that use the redundancy of the cyclic prefix (CP) have been developed [5,12-17]. The symbol synchronization algorithm [5] adopts the maximum-likelihood (ML) approach. However, being assumed in additive white Gaussian noise (AWGN) channels, the algorithm estimates the center of mass of the channel intensity profile 
rather than locating the first arriving path when used in multipath channels. One work [12] carries out coarse symbol timing synchronization in multipath channels, utilizing a correlation length that equals the summation of the channel and CP lengths. To make the algorithm more practical for synchronization, the simplified algorithm in [12] uses the correlation length that is equivalent to twice the CP length, subsequently degrading the performance. Despite the ability to identify the ISI-free region in multipath channels, an approach in that work [13] may require many symbols to obtain an accurate symbol timing estimate. The computation complexity of the rank method in another study [14] is high and can be incorporated in continuous-transmission networks. Yet, the performance of another method [15] may be disturbed by the CFO. A discrete stochastic approximation algorithm (DSA) for adaptive time synchronization has been developed in [16]. A related work [17] describes a maximum-likelihood (ML) approach. Although ML estimation methods produce better performance than ad hoc algorithms and can perform close to the theoretical Cramér-Rao lower bound (CRLB) on the mean square error, and their complexity is typically considered to be very high. Other works $[18,19]$ use either (blind) data [18] or frequency-domain pilots [19].

Conventional CP-based timing synchronization schemes may underper-form preamble-based ones. Despite the repeated structure in $\mathrm{CP}$, its correlation cannot be designed to resemble the impulse shape of training symbols. Therefore, the complexity of CP-based synchronization should be considerably increased to enhance its performance; otherwise, training symbols that can reduce the bandwidth efficiency should be used. This work thus develops a new synchronization scheme (considering multipath channels) having comparable complexity $(\sim O(N))$ to that of the simplest approach in [5] (considering AWGN channels), where $N$ denotes the number of subcarriers. As is well known, the channel length is related to the best symbol timing. However, this information is seldom used in literature. Conversely, to enhance the performance of symbol synchronization, the channel length is used explicitly in this work.

This work presents a new synchronization algorithm, assisted by channel length information, for OFDM systems based on the redundancy of CP. ISI significantly increases with symbol timing error in multipath fading channels $[1,2]$. Due to the characteristics of ISI, a new timing function, whose value is proportional to the interference, is developed. Of priority concern is how to locate the symbol timing estimate in the middle of the ISI-free region, because SINR of the received signals includes no penalty in this region. The proposed approach increases the robustness of the proposed algorithm because only phase rotation is introduced in the ISI-free region, which can be simply compensated by using a single-tap equalizer. Simulation results demonstrate that the maximum value of this function is at the correct timing offset, when the signal-to-noise ratio (SNR) is high or the signal power induced by the channel tap exceeds the noise power. Since random fluctuation of the timing function is unavoidable, the channel length is also determined to assist in locating the symbol timing at the middle of the ISI-free region. The proposed method is a 2-D function of the symbol timing offset and channel length. To reduce the complexity, i.e., search time and computation complexity, this work also develops an efficient search algorithm. Although ad hoc, the proposed timing function is demonstrated to be efficient because a complexity $\sim O$ $(N)$ and significant performance improvement are achieved.

The rest of this paper is organized as follows. Section II introduces the OFDM signal model and its correlation characteristics in multipath fading channels. Section III presents the proposed channel length assisted symbol synchronization algorithm. Section IV discusses in detail the design issues. Section V demonstrates simulation results. Finally, Section VI draws conclusions.

\section{OFDM signal model and correlation characteristics}

In wireless communications, the received signals are subjected to reflection and scattering from natural and man-made objects. Such phenomena result in the arrival of time variant multiple versions (multipaths) of transmitted signals at the receiving antenna. In a properly designed OFDM system, the CP length is normally longer than the channel length. The time-domain correlation characteristics of separated-by- $N$ data are thus related to neighboring symbols. In the following discussion, the signal model considers three consecutive symbols, i.e., the previous, the current, and the next symbols.

Let $h(l)$ denote the impulse response of multipath channels with $(L+1)$ uncorrelated taps. Consider an OFDM with $N$ subcarriers. The complex data are modulated onto the $N$ subcarriers via the inverse discrete Fourier transform (IDFT). CP of length ${ }^{N} G$ is inserted at the beginning of each OFDM symbol to prevent ISI and preserve the mutual orthogonality of sub-carriers.

Following parallel-to-serial conversion, the current OFDM symbol $x(n) ; n \in\left\{0,1, \ldots, N+N_{G}-1\right\}$, is finally transmitted through a multipath channel $h(l)$. Due to $\mathrm{CP}$, the transmitted data have the following property: if $n_{2} \neq n_{1}$ and $n_{2} \neq n_{1}+N, E\left[x\left(n_{1}\right) x^{*}\left(n_{2}\right)\right]=0$; otherwise, $E\left[x\left(n_{1}\right) x^{*}\left(n_{2}\right)\right]=\sigma_{x}^{2}$, where $\sigma_{x}^{2} \equiv E\left[|x(n)|^{2}\right]$ denotes the signal power. 
At the receiver, considering the previous OFDM symbol $x^{\prime}(n)$ of the current symbol, which is confined within $n \in\left\{-N-N_{G},-N-N_{G}+1 ; \ldots,-1\right\}$, the received data $\tilde{x}(n)$ can be written as

$$
\begin{aligned}
\tilde{x}(n) & =e^{j \frac{2 \pi n \varepsilon}{N}}\left(\sum_{l=0}^{L} h(l) x^{\prime}(n-l-\theta)+\sum_{l=0}^{L} h(l) x(n-l-\theta)\right)+w(n), \\
n & =\left\{\theta, \theta+1, \ldots, \theta+N+N_{G}-1\right\}
\end{aligned}
$$

where $\varepsilon$ denotes the normalized CFO; $\theta$ denotes the timing offset to be estimated, $\theta \in\left\{0,1, \ldots, N+N_{G}-1\right\}$, and $w(n)$ represents AWGN with zero-mean and variance $\sigma_{w}^{2}$. Notably, the channel length is assumed to be shorter than the CP length such that only partial CP of the current symbol is corrupted by the previous symbol. The ISI-free region is therefore attained in $n \in\{\theta+L, \theta$ $\left.+L+1, \ldots, \theta+N_{G}\right\}$.

Next, $\tilde{x}(n+N), n \in\left\{\theta, \theta+1, \ldots, \theta+N+N_{G}-1\right\}$, should be obtained to derive the correlation characteristics of separated-by- $N$ data. Similar to (1), while considering the following OFDM symbol $x^{\prime \prime}(n)$ of the current symbol, which is confined within $n \in\left\{N+N_{G}, N+N_{G}\right.$ $\left.+1, \ldots, 2\left(N+N_{G}\right)-1\right\}$, the received data $\tilde{x}(n+N)$ can be written as

$$
\begin{aligned}
\tilde{x}(n+N)= & e^{j \frac{2 \pi(n+N) \varepsilon}{N}}\left(\sum_{l=0}^{L} h(l) x(n+N-l-\theta)+\sum_{l=0}^{L} h(l) x^{\prime \prime}(n+N-l-\theta)\right) \\
& +w(n+N), \quad n=\left\{\theta, \theta+1, \ldots, \theta+N+N_{G}-1\right\} .
\end{aligned}
$$

Determination of the correlation characteristics is simplified using the models in (1) and (2). Since $x(n) ; x^{\prime}(n)$; $x^{\prime \prime}(n) ; h(l)$, and $w(n)$ are mutually uncorrelated, the correlation between $\tilde{x}(n)$ and $\tilde{x}(n+N)$ can be expressed as (see Appendix A)

$$
E\left[\tilde{x}(n) \tilde{x}^{*}(n+N)\right]= \begin{cases}\sigma_{x}^{2} e^{-j 2 \pi \varepsilon} \sum_{l=0}^{n-\theta}|h(l)|^{2}, & n \in I_{1} \\ \sigma_{x}^{2} e^{-j 2 \pi \varepsilon} \sum_{l=0}^{L}|h(l)|^{2}, & n \in I_{2} \\ \sigma_{x}^{2} e^{-j 2 \pi \varepsilon} \sum_{l=n-\theta-N_{G}+1}^{L}|h(l)|^{2}, & n \in I_{3} \\ 0, & n \in I_{4}\end{cases}
$$

where $I_{1} \equiv\{\theta, \theta+1, \ldots, \theta+L-1\}, I_{2} \equiv\{\theta+L, \theta+L+$ $\left.1, \ldots, \theta+N_{G}-1\right\}, I_{3} \equiv\left\{\theta+N_{G}, \theta+N_{G}+1, \ldots . \theta+N_{G}+L\right.$ - 1$\}$, and $I_{4} \equiv\left\{\theta+N_{G}+L, \theta+N_{G}+L+1, \ldots, \theta+N+\right.$ $\left.N_{G}-1\right\}$. Notably, no assumption is made regarding the transmitted data.

Figure 1 illustrates the correlation (3) for $\varepsilon=0$. Its shape depends on the channel condition. Nonzero correlation values of separated-by- $N$ samples are attributed to CP. Due to linear convolution of the transmitted data with a channel, the length of nonzero correlation values is $N_{G}+L$.

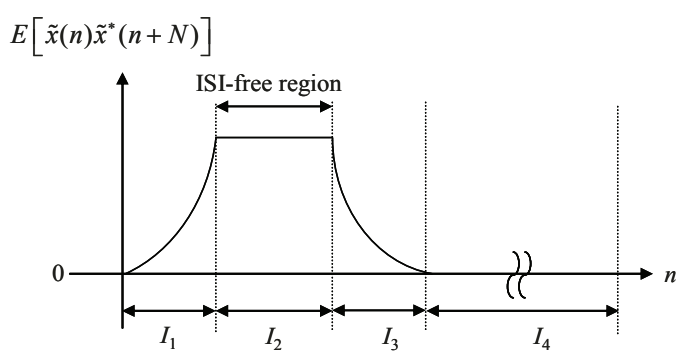

Figure 1 Correlation characteristics of received separated-by- $N$ data.

Taking the magnitude of (3) yields,

$$
\left|E\left[\tilde{x}(n) \tilde{x}^{*}(n+N)\right]\right|=\left\{\begin{array}{ll}
\sigma_{x}^{2} \sum_{l=0}^{n-\theta}|h(l)|^{2}, & n \in I_{1} \\
\sigma_{x}^{2} \sum_{l=0}^{L}|h(l)|^{2}, & n \in I_{2} \\
\sigma_{x}^{2} \sum_{l=n-\theta-N_{G}+1}^{L}|h(l)|^{2}, & n \in I_{3} \\
0, & n \in I_{4}
\end{array} .\right.
$$

Notably, the correlation results in $I_{2}$ (ISI-free region) with a plateau are greater than those in $I_{1}$ and $I_{3}$. Equation 4 can be regarded as the desired signal power. Similarly,

$$
E\left[|\tilde{x}(n)|^{2}\right]=\sigma_{x}^{2} \sum_{l=0}^{L}|h(l)|^{2}+\sigma_{w^{\prime}}^{2} \quad \forall n \in I
$$

where $I=I_{1} \cup I_{2} \cup I_{3} \cup I_{4}$. Therefore, (5) is the signal power plus the AWGN power. These characteristics are exploited in the following section.

\section{Proposed symbol synchronization}

\section{A Channel length assisted symbol synchronization}

Before the synchronization algorithm is introduced, it should be noted that the cross-correlation result in $I 2$ denotes the signal power (without ISI and noise), which comprises the power spread by multipath channels. The loss and leakage of the desired signal power in $I 1$ and $I 3$, respectively, are caused by ISI. Evidently, the autocorrelation result is the total signal power. Based on (4) and (5), a new timing function is obtained

$$
\begin{aligned}
& \Lambda(k, m) \equiv-\psi^{2}(k, m)+2\left(N_{G}-m\right) \psi(k, m) \sigma_{w}^{2} \\
& \quad k \in\left\{0,1, \ldots, N+N_{G}-1\right\} \text { and } m \in\left\{0,1, \ldots, N_{G}-1\right\}
\end{aligned}
$$


where

$$
\begin{aligned}
\psi(k, m)= & \frac{1}{2} \sum_{n=m}^{N_{G}-1}\left(E\left[|\tilde{x}(n+k)|^{2}\right]+E\left[|\tilde{x}(n+N+k)|^{2}\right]\right) \\
& -\left|\sum_{n=m}^{N_{G}-1} E\left[\tilde{x}(n+k) \tilde{x}^{*}(n+N+k)\right]\right| .
\end{aligned}
$$

The timing function is 2-D and is generated by sliding windows with all possible channel lengths (ranging from one to $N_{G}$ ) at all possible sampling points in an OFDM symbol. The end of this subsection describes the rationale for the timing function. Before doing so, some properties of the timing function are introduced first.

The proposed timing function has the following properties.

Property 1 The function,

$$
\phi(n)=\frac{1}{2}\left(E\left[|\tilde{x}(n)|^{2}\right]+E\left[|\tilde{x}(n+N)|^{2}\right]\right)-\left|E\left[\tilde{x}(n) \tilde{x}^{*}(n+N)\right]\right|, n \in I,
$$

has a minimum plateau in the ISI-free region.

Proof: Inserting (4) and (5) into (8) yields,

$$
\phi(n)= \begin{cases}\sigma_{x}^{2}\left(\sum_{l=0}^{L}|h(l)|^{2}-\sum_{l=0}^{n-\theta}|h(l)|^{2}\right)+\sigma_{w^{\prime}}^{2} & n \in I_{1} \\ \sigma_{x}^{2}\left(\sum_{l=0}^{L}|h(l)|^{2}-\sum_{l=0}^{L}|h(l)|^{2}\right)+\sigma_{w^{\prime}}^{2} & n \in I_{2} \\ \sigma_{x}^{2}\left(\sum_{l=0}^{L}|h(l)|^{2}-\sum_{l=n-\theta-N_{G}+1}^{L}|h(l)|^{2}\right)+\sigma_{w}^{2}, n \in I_{3} & n \in I_{4} \\ \sigma_{x}^{2} \sum_{l=0}^{L}|h(l)|^{2}+\sigma_{w^{\prime}}^{2} & \\ = & \begin{cases}\sigma_{x}^{2} \sum_{l=n-\theta+1}^{L}|h(l)|^{2}+\sigma_{w^{\prime}}^{2}, & n \in I_{1} \\ \sigma_{w}^{2}{ }_{n-\theta-N_{G}} & n \in I_{2} \\ \sigma_{x}^{2} \sum_{l=0}|h(l)|^{2}+\sigma_{w}^{2}, n \in I_{3} & \\ \sigma_{x}^{2} \sum_{l=0}^{L}|h(l)|^{2}+\sigma_{w^{\prime}}^{2} & n \in I_{4}\end{cases} \end{cases}
$$

Owing to the multipath fading channels, $\varphi(n)$ apparently has a minimum plateau with a value of $\sigma_{w}^{2}$ in the ISI-free region. The proof follows.

Property 2 The maximum value of the timing function (6) occurs at $k=\theta$ and $m=L$ under the conditions of $\sigma_{x}^{2}|h(0)|^{2}>\sigma_{w}^{2}$ and $\sigma_{x}^{2}|h(0)|^{2}>\sigma_{w}^{2}$. (The signal power induced by the channel tap is larger than the AWGN power.)

Proof: First, the following function $\varphi^{\prime}(n)$ is shown to be positive in the ISI-free region and negative in the ISI regions, when $\sigma_{x}^{2}|h(L)|^{2}>\sigma_{w}^{2}$ and $\sigma_{x}^{2}|h(0)|^{2}>\sigma_{w}^{2}$ are satisfied. Based on property 1 ,

$$
\begin{aligned}
\phi^{\prime}(n) & \equiv-\phi(n)+2 \sigma_{w}^{2} \\
& =\left\{\begin{array}{ll}
-\sigma_{x}^{2} \sum_{l=n-\theta+1}^{L}|h(l)|^{2}+\sigma_{w^{\prime}}^{2} & n \in I_{1} \\
\sigma_{w^{\prime}}^{2} & n \in I_{2} \\
-\sigma_{x}^{2} \sum_{l=0}^{n-\theta-N_{G}}|h(l)|^{2}+\sigma_{w}^{2}, & n \in I_{3} \\
-\sigma_{x}^{2} \sum_{l=0}^{L}|h(l)|^{2}+\sigma_{w^{\prime}}^{2} & n \in I_{4}
\end{array} .\right.
\end{aligned}
$$

From (10), $\varphi^{\prime}(n)$ is a positive constant, $\sigma_{w}^{2}$, in $I_{2}$. In $I_{1}$, $\varphi^{\prime}(n)$ is a strictly increasing function with a maximum of $-\sigma_{x}^{2}|h(L)|^{2}+\sigma_{w}^{2}$ at $n=\theta+L-1$. Therefore, when $\sigma_{x}^{2}|h(L)|^{2}>\sigma_{w}^{2}$, the values of (10) in $I_{1}$ are all negative. Similarly, when $\sigma_{x}^{2}|h(0)|^{2}>\sigma_{w}^{2}$, the values of $\varphi^{\prime}(n)$ in $I_{3}$ and $I_{4}$ are all negative. The functions, $\varphi(n)$ (in property $1)$ and $\varphi^{\prime}(n)$, are used to prove property 2 .

The correlation (3) generally has a complex value, which is introduced owing to the CFO. The phase rotations of all correlations are the same at all sampling points, which can be eliminated by the absolute operation. Additionally, $\sigma_{x}^{2}$ and $|h(l)|^{2}$ are larger than 0 ; therefore,

$$
\left|\sum_{n=m}^{N_{G}-1} E\left[\tilde{x}(n+k) \tilde{x}^{*}(n+N+k)\right]\right|=\sum_{n=m}^{N_{G}-1}\left|E\left[\tilde{x}(n+k) \tilde{x}^{*}(n+N+k)\right]\right| .
$$

Equations 7 and 8 yield $\psi(k ; m)$ as

$$
\psi(k, m)=\sum_{n=m}^{N_{G}-1} \phi(n+k) .
$$

Moreover, the timing function (6) can be written as

$$
\Lambda(k, m)=\psi(k, m)\left[-\psi(k, m)+2\left(N_{G}-m\right) \sigma_{w}^{2}\right] .
$$

Therefore, via (10) and (12), $\Lambda(k, m)$ can be expressed as

$$
\Lambda(k, m)=\left(\sum_{n=m}^{N_{G}-1} \phi(n+k)\right)\left(\sum_{n=m}^{N_{G}-1} \phi^{\prime}(n+k)\right) .
$$

From the above equation, since $\varphi(n)>0, \varphi^{\prime}(n)$ is a positive constant in the ISI-free region and is negative in the ISI regions, $\Lambda(k, m)$ has a maximum value at $(k$; $m)=(\theta, L)$ (see Appendix B). The proof follows.

Property 2 can be relaxed for high SNRs, as described by the following property.

Property 3 For high SNRs, the maximum value of the timing function (6) occurs at $k=\theta$ and $m=L$ (without the constraint that the signal power induced by the channel tap should be larger than the AWGN power). 
Proof: When the SNR is high,

$$
\phi(n) \approx \begin{cases}\sigma_{x}^{2} \sum_{l=n-\theta+1}^{L}|h(l)|^{2}, & n \in I_{1} \\ \sigma_{w^{\prime}}^{2} & n \in I_{2} \\ \sigma_{x}^{2} \sum_{l=0}^{n-\theta-N_{G}}|h(l)|^{2}, & n \in I_{3} \\ \sigma_{x}^{2} \sum_{l=0}^{L}|h(l)|^{2}, & n \in I_{4}\end{cases}
$$

and

$$
\phi^{\prime}(n) \approx\left\{\begin{array}{ll}
-\sigma_{x}^{2} \sum_{l=n-\theta+1}^{L}|h(l)|^{2}, & n \in I_{1} \\
\sigma_{w^{\prime}}^{2} & n \in I_{2} \\
-\sigma_{x}^{2} \sum_{l=0}^{n-\theta-N_{G}}|h(l)|^{2}, & n \in I_{3} \\
-\sigma_{x}^{2} \sum_{l=0}^{L}|h(l)|^{2}, & n \in I_{4}
\end{array} .\right.
$$

Therefore, $\varphi^{\prime}(n)$ appears to be a positive constant $\sigma_{w}^{2}$ in $I_{2}, \varphi^{\prime}(n)$ is negative in $I_{1}, I_{3}$ and $I_{4}$. With (15) and (16), the timing function (14) obviously has a maximum value of $\left(N_{G}-L\right)^{2} \sigma_{w}^{4}$ at $k=\theta$ and $m=L$. The proof follows.

Based on Properties 2 and 3, the timing offset $\theta$ and the channel length $L$ are estimated according to the maximum point of the timing function $\Lambda(k ; m)$ :

$$
(\hat{\theta}, \hat{L})=\arg \max _{k} \max _{m} \Lambda(k, m) .
$$

Finally, since no penalty applies when the symbol timing is located in the ISI-free region, given the inevitable random fluctuation in the estimate, the preferred strategy of the synchronization is to locate the symbol timing in the middle of the ISI-free region. Via the information of the first sample of $I_{2}$, i.e., $\hat{\theta}+\hat{L}$, and the length of the ISI-free region $N_{G}-\hat{L}$, the final estimate of symbol timing, $\hat{\theta}_{o}$, is located in the middle position of the ISI-free region as

$$
\begin{aligned}
\hat{\theta}_{o} & =\hat{\theta}+\hat{L}+\left\lfloor\frac{N_{G}-\hat{L}}{2}\right\rfloor \\
& =\hat{\theta}+\frac{N_{G}}{2}+\left\lceil\frac{\hat{L}}{2}\right\rceil
\end{aligned}
$$

where $N_{G}$ is assumed to be an even number, $\lfloor\cdot\rfloor$ and $\lceil .7$ denote the floor and ceiling functions, respectively.

Notably, when the symbol timing is located in the ISIfree region, only phase rotation is introduced, which can be simply compensated for by using a single-tap equalizer. Additionally, Property 3 indicates that an accurate estimate can be obtained when the SNR is high. Based on Property
2, from low to moderate SNRs, an accurate estimate can also be obtained if $\sigma_{x}^{2}|h(L)|^{2}>\sigma_{w}^{2}$ and $\sigma_{x}^{2}|h(0)|^{2}>\sigma_{w}^{2}$ are satisfied. If the above-mentioned conditions are not satisfied, $\hat{\theta}$ will typically be around $\theta$, because $\varphi^{\prime}(n)$ is a strictly increasing/decreasing function in $I_{1} / I_{3}$. Besides, due to the SINR plateau, tolerance is allowed if the final estimate of symbol timing $\hat{\theta}_{o}$ lies in the ISI-free region. In this condition, the channel length estimate assists in locating $\hat{\theta}_{o}$ in the middle position of ISI-free region, thus increasing the estimation accuracy. Hence, the proposed method utilizes the plateau in the ISI-free region.

Following the introduction of the proposed timing function and its properties, its design rationale is brie $y$ described here. The core function, $\varphi(n)$, expressed in (8) is proportional to the incurred interference. By using a simple algebraic equation, $\varphi^{\prime}(n)$ is obtained from $\varphi(n)$. Then, by considering all possible lengths of the ISI-free region, the timing function $\Lambda(k, m)$ (14) is expressed by $\varphi(n)$ and $\varphi^{\prime}(n)$ and can be further simplified as (6).

\section{B Search algorithm}

To prevent the timing function from fluctuating when $m$ is small and reduce the computation complexity of the 2-D search algorithm (17), this work presents and implements an algorithm, as described in Table 1 . In the proposed algorithm, $\Upsilon_{\max }(m=M)$ denotes the maximum value when $m$ increases from 0 to $M, M \in\{0,1, \ldots$, $\left.N_{G}-1\right\} ; \Theta_{\max }(k)$ denotes the maximum value of all $k$ at a given $m$, and -maxValue denotes the smallest negative value that a computer can represent.

When $N_{G}-m$ exceeds the length of the ISI-free region, based on Property $2, \Upsilon_{\max }(m)$ is smaller than that of the global maximum value at $m=L$. The value of $\Upsilon_{\max }(m)$ increases until $N_{G}-m$ equals the length of the ISI-free region. $\Upsilon_{\max }(m)$ starts to decline, when $N_{G}$ $m$ decreases continuously and eventually becomes smaller than the ISI-free region length.

Based on Property 3, for high SNR conditions, Figure 2 presents the timing function, $\Lambda(k, m)$, as a function of $m$, i.e., $\Upsilon_{\max }(m)$. For clarity, this figure labels only some values of $\Upsilon_{\max }(m)$ at $k=\theta$. The values of $\Upsilon_{\max }(m)$ at other samples can be similarly shown and found to be smaller than that at $(k, m)=(\theta, L)$. According to this figure, for increasing $m$ when $m \in\left\{L, L+1, \ldots, N_{G}-1\right\}$, $\Upsilon_{\max }(m)$ declines with the factor $\left(N_{G}-m\right)^{2}$. For decreasing $m$ when $m \in\{0,1, \ldots, L-1\}, \Upsilon_{\max }(m)$ declines proportionally according to $-\left(\sum_{l=0}^{L-m-1}|h(l)|^{2}\right)^{2}$.

\section{Implementation issues}

\section{A Auto- and cross-correlations}

The timing function (6) requires theoretical auto- and cross-correlations, which are often realized using the 
Table 1 2-D search algorithm

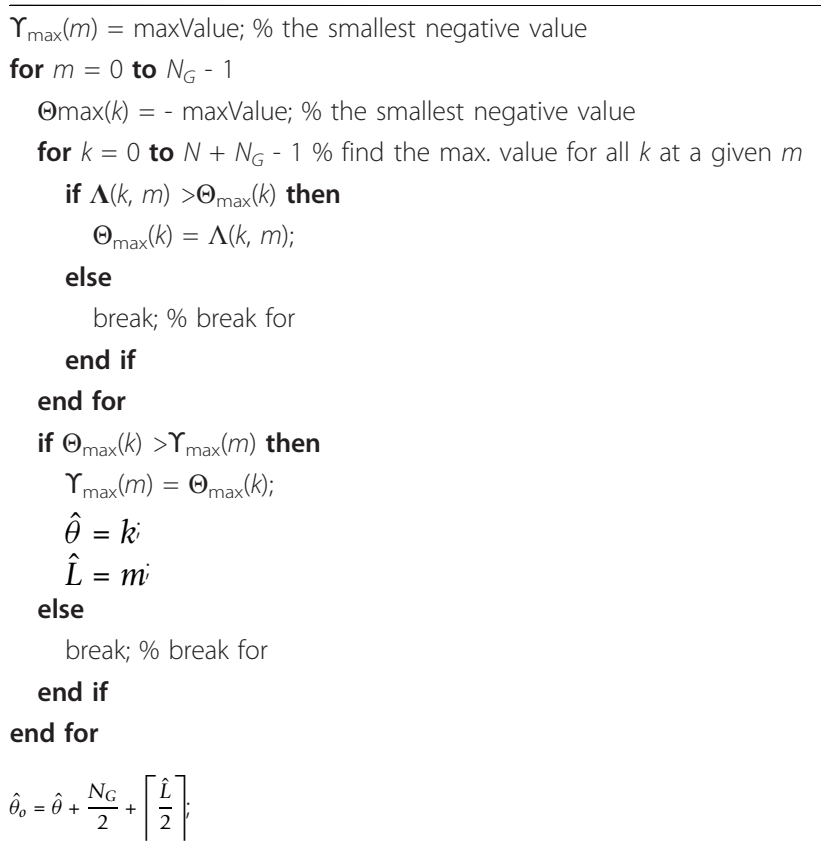

sample correlation. When $N$ is large, the sampled data $\tilde{x}(n)$ can be modeled approximately as complex Gaussian using the central limit theorem. Therefore, the sample auto- and cross-correlations can be obtained by averaging all of the symbols.

\section{B Computation complexity}

Since the proposed algorithm may terminate before searching for all possible combinations of $k$ and $m$, the worst-case complexity (for all $k$ and $m$ ) is evaluated. The sampled correlation realizations of (4) and (5) both require $N+N_{G}$ complex multiplications. The timing function (6) requires additional $3\left(N+N_{G}\right)$ real multiplications. A real multiplication roughly costs $1 / 4$ complex multiplication. In summary, the total number of required complex multiplications of the proposed symbol synchronization is $\left(N+N_{G}\right)(3+3 / 4)=3: 75(N+$ $\left.N_{G}\right)$. The number of required complex additions of the proposed symbol synchronization in (7) is $1.5 N_{G}(N+$ $N G)(N G+1)$.

Since the complexity of an addition is substantially less than that of a multiplication, the proposed method has a worst-case complexity of approximately $3.75 / 3=$ 1:25 times that of the representative and simplest algorithm [5]. In other cases, complexity of the proposed method may be lower since its complexity depends on the channel length. Moreover, according to our results, the proposed method outperforms conventional methods, as presented in the next section.

\section{Simulation results}

Monte Carlo simulations are conducted to evaluate the performance of the estimators. An OFDM system with $N=128$ and $N_{G}=16$ is considered. The simulated modulation scheme is QPSK. The signal bandwidth is $2.5 \mathrm{MHz}$, and the radio frequency is $2.4 \mathrm{GHz}$. The subcarrier spacing is $19.5 \mathrm{kHz}$. The OFDM symbol duration is $57.6 \mu \mathrm{s}$. The simulations are evaluated under the effect of the $\mathrm{CFO}=33.3 \%$ subcarrier spacing, i.e., 6.5 $\mathrm{kHz}$. To verify the performance of the proposed technique, channel length is assumed to be uniformly distributed within the range of $\left[1, \ldots, N_{G}-1\right]$. In each simulation run, the channel taps are randomly generated by using independent zero-mean unit-variance complex Gaussian variables with $\sum_{l}|h(l)|^{2}=1$. Namely, the power of channel taps is normalized to one. In each run, 20 OFDM symbols are tested. Metrics of the proposed and

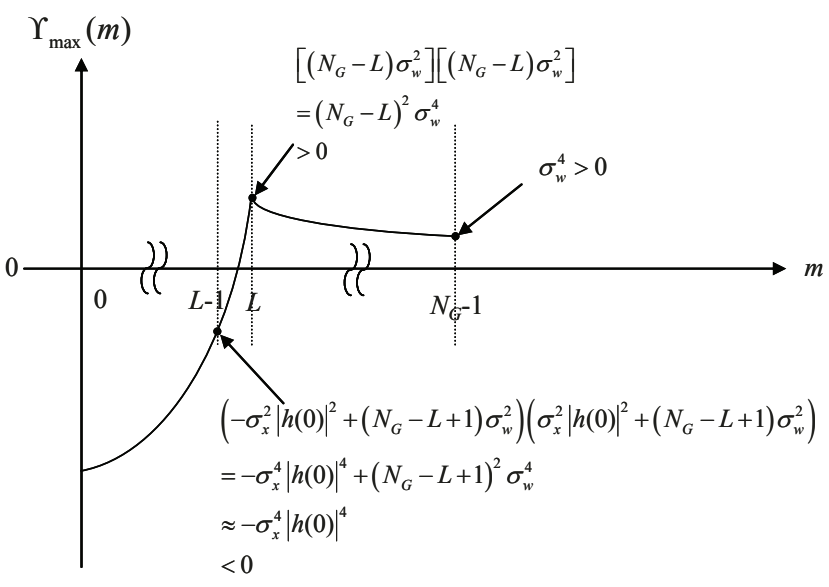

Figure 2 Timing function $\Upsilon_{\max }(m)$ as a function of $m$ 
compared estimators are averaged over simulated symbols.

\section{A MSE of symbol synchronization in multipath channels} Performance of the symbol synchronization is evaluated by the estimators' normalized mean-squared error (MSE) by $N^{2}$, i.e., the MSE is defined as ${ }^{\mathrm{a}}$

$$
\mathrm{MSE} \equiv \begin{cases}E\left\{\left[\frac{\hat{\theta}_{o}-(\theta+L)}{N}\right]^{2}\right\}, & \hat{\theta}_{o}<\theta+L \\
E\left\{\left[\frac{\hat{\theta}_{o}-\left(\theta+N_{G}-1\right)}{N}\right]^{2}\right\} & \begin{array}{l}
\hat{\theta}_{o}>\theta+N_{G}-1 \\
\\
0, L \leq \hat{\theta}_{o} \leq \theta+N_{G}-1
\end{array} .\end{cases}
$$

Notably, the expectation of estimate in (19) is replaced by its average over all simulation results. The proposed estimator is compared with the ML estimator [5], MMSE estimator [12], and Blind estimator [14]. MSE of the estimate is plotted as a function of the SNR. The SNR is defined as

$$
\mathrm{SNR} \equiv \frac{\sigma_{x}^{2} \sum_{l}|h(l)|^{2}}{\sigma_{w}^{2}}=\frac{\sigma_{x}^{2}}{\sigma_{w}^{2}} .
$$

The noise variance used in this work and in [5] can be estimated by [20] which is beyond the scope of this paper. In the simulations, $\sigma_{w}^{2}$ is assumed to be perfectly known.

Figure 3 plots the MSE of the estimated symbol timing as a function of SNR in various multipath fading channels. The performance is averaged over 10,000 channel realizations. As shown, the proposed estimator achieves a lower MSE than the compared estimators, especially at high SNRs. This finding demonstrates that in addition to its robustness against variation in multipath fading channels, the proposed algorithm can significantly reduce MSE more than the estimators [5,12,14]. Although not shown here for brevity, among all of the compared estimators, the method in [5] performs the best in the AWGN channel.

Next, the channel model of the ITU-R vehicular B channel [21] is considered to investigate how the proposed method performs in a standard multipath fading channel. The adopted channel has the following 6 taps,

$$
\begin{aligned}
& \text { Channel } 2(L=5) \text { with channel tap powers (in } \mathrm{dB}) \text { : } \\
& \begin{aligned}
& {[-2.5(0 \mathrm{~ns}) \quad 0(300 \mathrm{~ns})-12.8(8900 \mathrm{~ns})-10(12900 \mathrm{~ns})} \\
& -25.2(17100 \mathrm{~ns}) \quad-16(20000 \mathrm{~ns})] .
\end{aligned}
\end{aligned}
$$

Figure 4 plots the MSE of the estimated symbol timing as a function of SNR in the ITU-R vehicular B channel. Also plotted in this figure are the MSEs of the estimators in multipath fading channels for comparison. Since the estimator [14] generally has a better performance than the estimators $[5,12,14]$ in multipath channels, for clarity, only the performance of the estimator [14] is shown. The MSE of the proposed estimator in the selected channel is worse than that in the randomly generated multipath fading channels when SNR is low; however, when SNR is high, the relation reverses. This is unsurprising since the performance of the synchronization typically depends on the channel condition. Furthermore, the MSE of the proposed estimator declines with an increasing SNR, while that of the compared estimator improves only slightly.

\section{B MSE of symbol synchronization under the effect of CP length}

Since the proposed estimator is based on the CP, Figure 5 plots the MSE of the estimated symbol timing, under $N_{G}=16$ and $N_{G}=32$, as a function of SNR in multipath fading channels. For clarity, only the performance of the compared estimator [14] is shown. The MSE of the proposed estimator declines more rapidly than the compared estimator does with an increasing $N_{G}$ ' especially at high SNRs. Restated, the proposed estimator can perform as well as the other estimators, but with fewer received blocks. Importantly, the performance of the proposed estimator improves rapidly with an increasing $N_{G}$, further confirming the consistency of the proposed estimator.

\section{$C$ Bit error rate}

Figure 6 plots the bit error rate (BER) of the proposed estimator and the ML estimator [5], as a function of SNR in multipath fading channels. To focus on synchronization, the channel frequency response used for channel equalization is assumed to be perfectly known at the receiver. This figure also shows the BER for the case of perfect synchronization, indicating that, in comparison with perfect synchronization, BER performance loss is observed with symbol timing error, even under perfect channel estimation. This figure further reveals that the proposed estimator performs better than the compared estimator [5] in terms of BER. At a high SNR ( $\geq 25 \mathrm{~dB})$, the BER performance achieved by the proposed estimator approaches that with perfect synchronization. This is owing to that, at a high SNR, after compensation for the synchronization error by the proposed estimator, the effect of residual error is almost negligible.

\section{Conclusion}

This work has presented a novel channel length assisted synchronization scheme based on the properties of OFDM signals. Only simple operations, such as the multiplication and addition operations, are necessary. Simulation results demonstrate that the maximum value obtained by the proposed timing function is correct when the SNR is high. Otherwise, the correct timing 


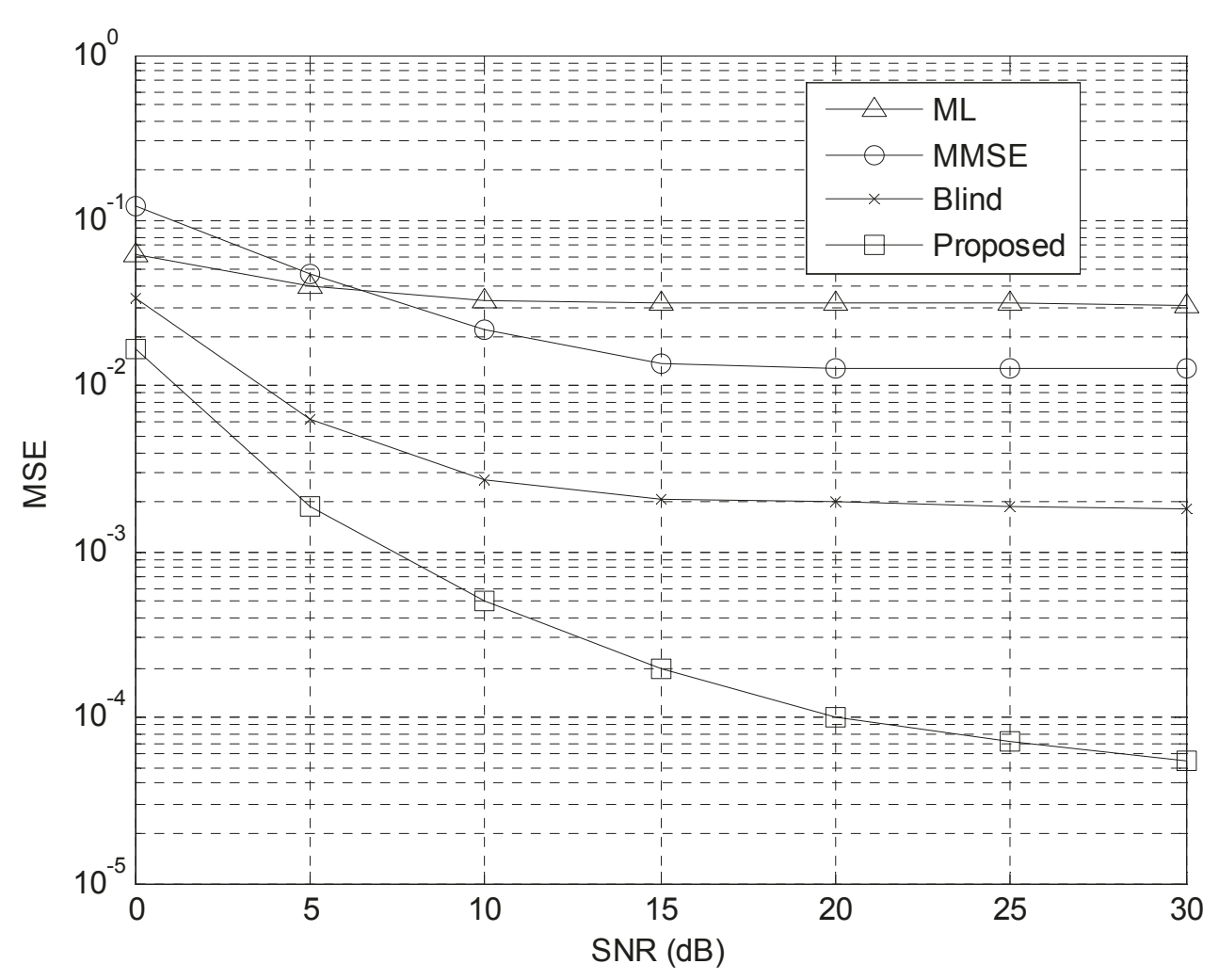

Figure 3 The MSE of the estimated symbol timing as a function of SNR in multipath fading channels

estimate is ensured when the signal power induced by the channel tap exceeds the noise power. This finding suggests that the channel tap can be identified when its induced signal power exceeds the AWGN power. This work also identifies the symbol timing in the middle of the ISI-free region by estimating the channel length through use of the proposed timing function. Simulation results verify that the proposed estimation markedly reduces the MSE of the symbol timing estimate in multipath fading channels.

\section{Appendix I}

The appendix presents detailed derivation of the correlation characteristics (3). With (1) and (2), since $x(n)$, $x^{\prime}(n) ; x^{\prime \prime}(n) ; h(l)$, and $w(n)$ are uncorrelated,

$$
\begin{aligned}
E\left[\tilde{x}(n) \tilde{x}^{*}(n+N)\right] & =e^{-j 2 \pi \varepsilon} \sum_{l_{1}=0}^{L} \sum_{l_{2}=0}^{L} E\left[h\left(l_{1}\right) h^{*}\left(l_{2}\right)\right] E\left[x\left(n-l_{1}-\theta\right) x^{*}\left(n+N-l_{2}-\theta\right)\right] \\
& =e^{-j 2 \pi \varepsilon} \sum_{l=0}^{L}|h(l)|^{2} E\left[x(n-l-\theta) x^{*}(n+N-l-\theta)\right] .
\end{aligned}
$$

Note that the correlations of transmitted separated-by$N$ data are nonzero only in the CP. That is, for

$$
0 \leq n-l-\theta \leq N_{G}-1 \text {. }
$$

Or, equivalently, (23) can be written as

$$
n-\theta-N_{G}+1 \leq l \leq n-\theta .
$$

Since $0 \leq l \leq L$, the correlation characteristics are nonzero for

$$
l \in\{0,1, \ldots, L\} \cap\left\{n-\theta-N_{G}+1, n-\theta-N_{G}+2, \ldots, n-\theta\right\} .
$$

With $\theta \leq n \leq \theta+N+N_{G}-1$, the correlation characteristics can be easily shown to be (3).

\section{Appendix II}

Given the characteristics of $\varphi(n)$ and $\varphi^{\prime}(n), \Lambda(k, m)$ can be written in a general form as

$$
\begin{aligned}
\Lambda(k, m) & =\left(\sum_{n=m}^{N_{G}-1} \phi(n+k)\right)\left(\sum_{n=m}^{N_{G}-1} \phi^{\prime}(n+k)\right) \\
& =[A(k, m)+B(k, m)][A(k, m)-B(k, m)] \\
& =A^{2}(k, m)-B^{2}(k, m)
\end{aligned}
$$

where $A(k, m) \geq 0$ and $B(k, m) \geq 0$, and their values depend on $k$ and $m$. For example, according to Figure 7 , for the first case when the summation is within the range of $I_{1}, A(k, m)=0$ and $B(k, m)>0$; and hence, $\Lambda(k$, 


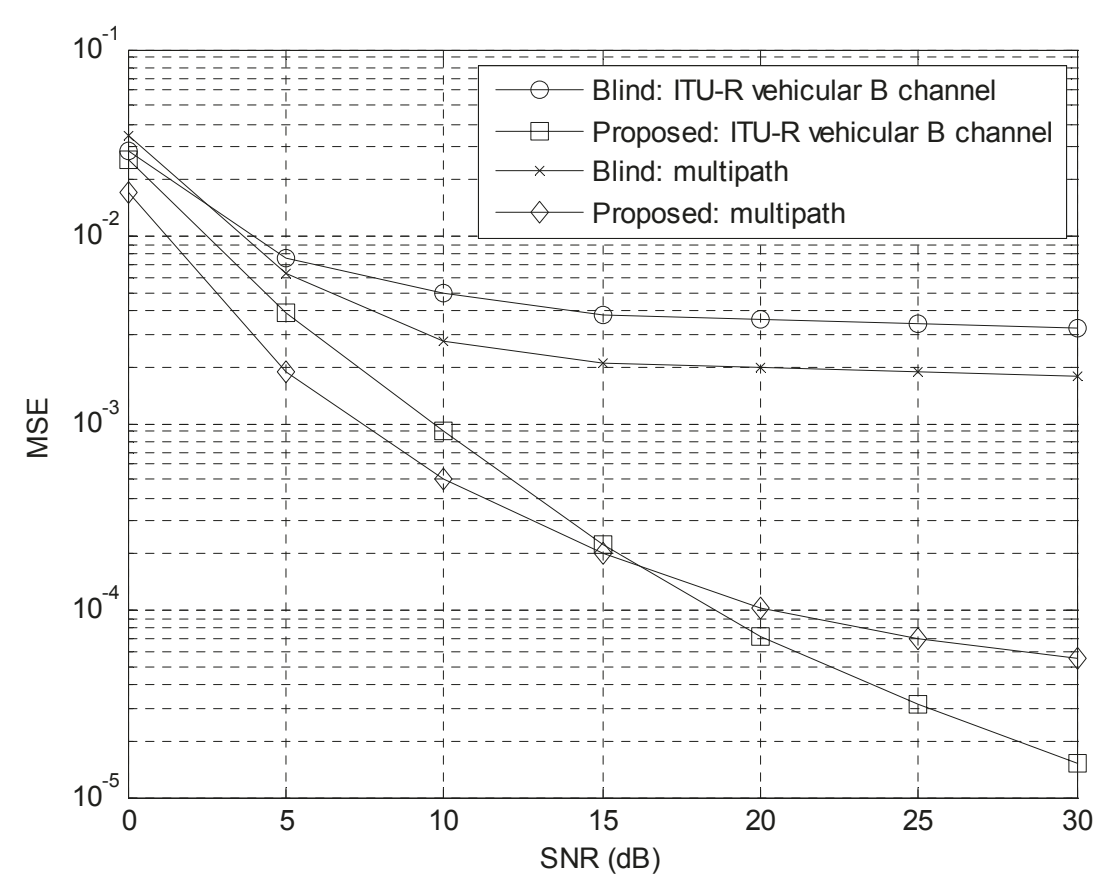

Figure 4 The MSE of the estimated symbol timing as a function of SNR in the ITU-R vehicular B channel.

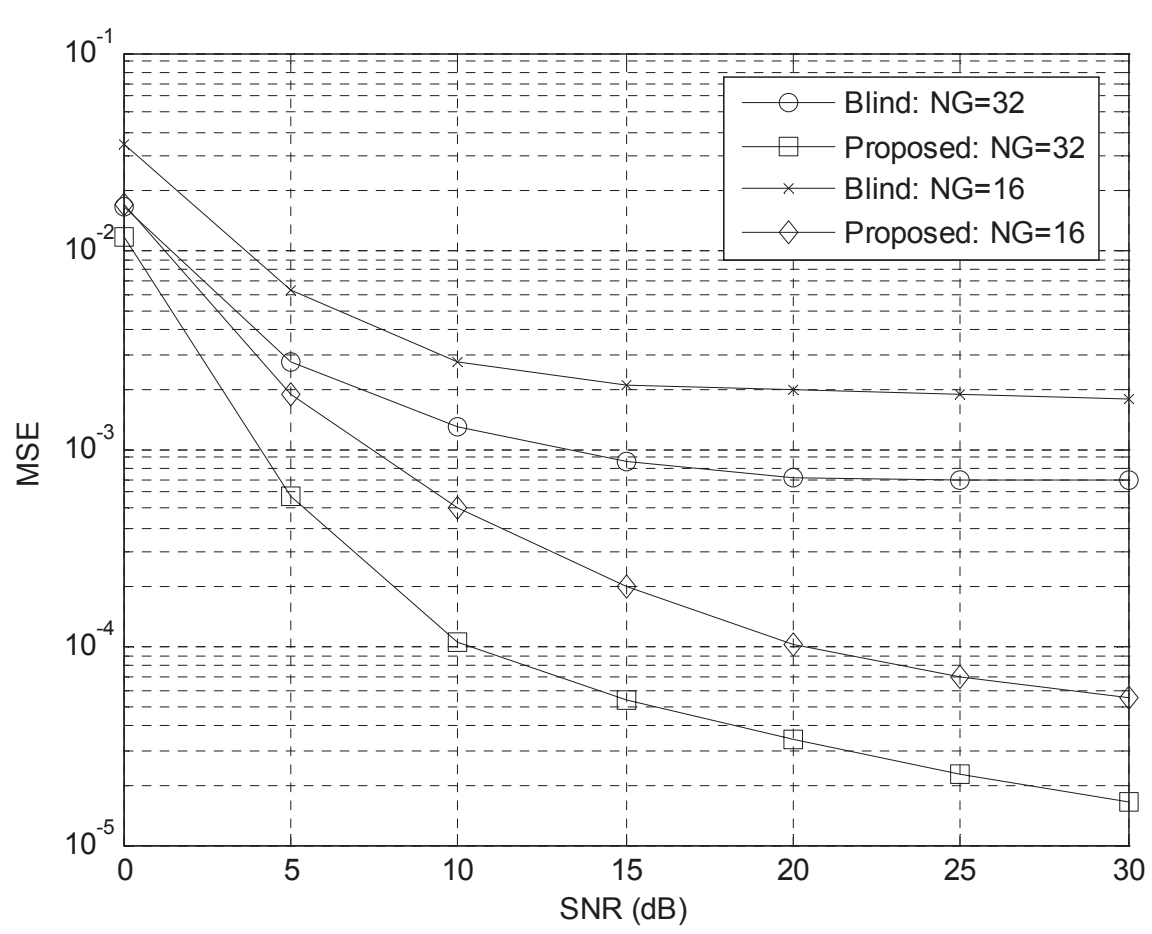

Figure 5 The MSE of the estimated symbol timing, under $N_{G}=16$ and $N_{G}=32$, as a function of SNR in multipath fading channels. 


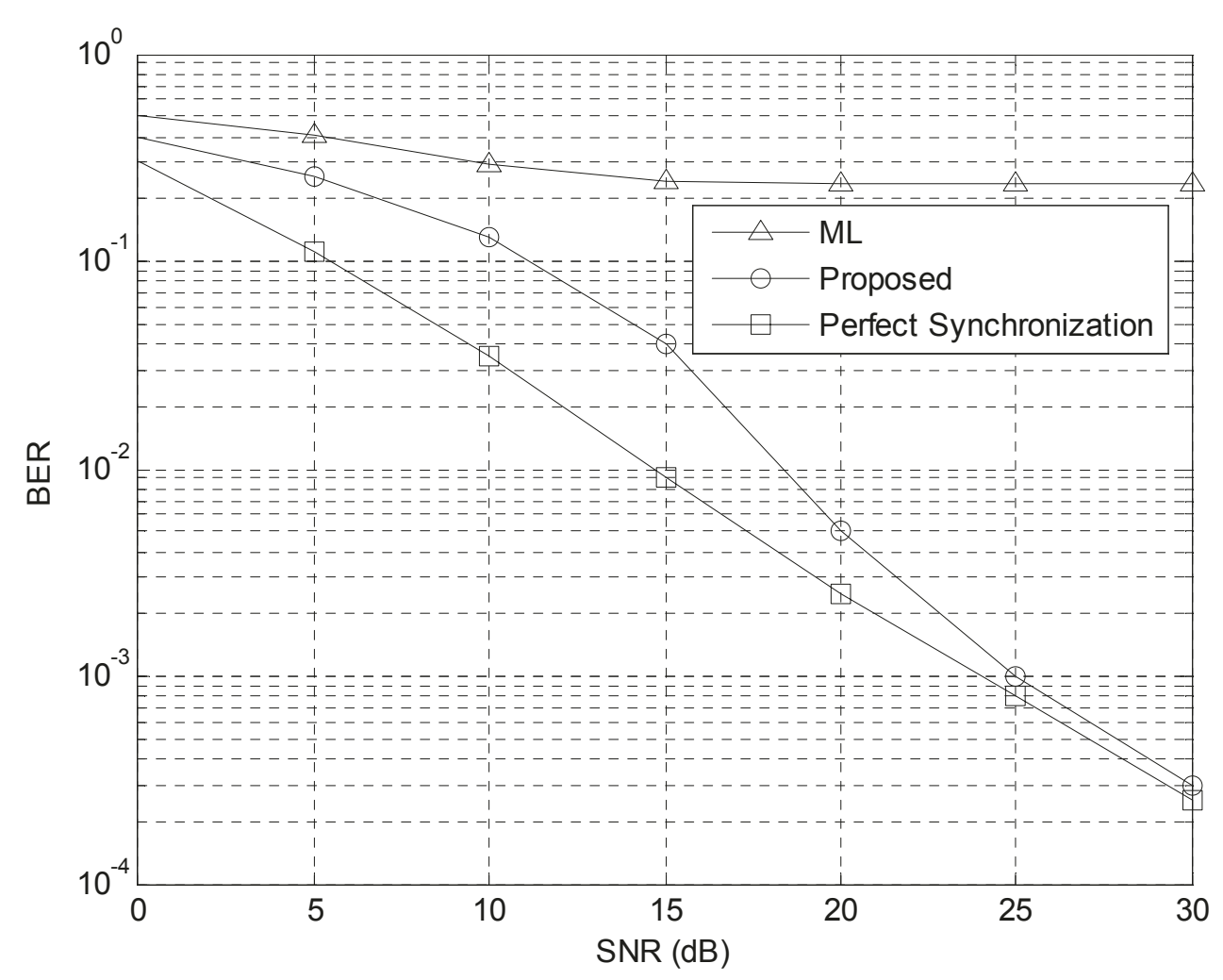

Figure 6 BER performance.

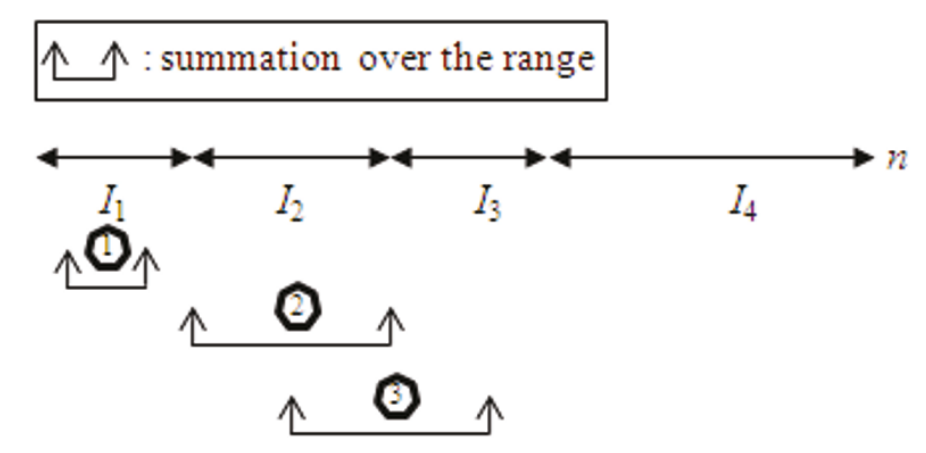

Figure 7 Three cases used to demonstrate the maximum of the proposed metric when $(k ; m)=(\theta, L)$

$m)<0$. For the second case in Figure 7, i.e., the summation encompasses the entire range of $I_{2}$, $A(k, m)=\left(N_{G}-L\right) \sigma_{w}^{2}$ and $B(k, m)=0$; therefore, $\Lambda(k, m)=\left(N_{G}-L\right)^{2} \sigma_{w}^{4}$, which is the maximum value. For the third case in Figure $7, A(k, m)<\left(N_{G}-L\right) \sigma_{w}^{2}$ and $B(k, m) \neq 0$, therefore, the value of $\Lambda(k, m)$ is smaller than that of case two. Generally, for all other cases except $(k, m)=(\theta, L), A(k, m)<\left(N_{G}-L\right) \sigma_{w}^{2}$ and $B(k$, $m) \geq 0$. Therefore, we conclude that $\Lambda(k, m)$ has its maximum value when $(k, m)=(\theta, L)$.

\section{Endnote}

${ }^{a}$ Since the SINR has a plateau in the ISI-free region that produces no penalty, the MSE is counted as zero when the estimate is located in the ISI-free region. Restated, MSE represents the distance from the estimated symbol timing to the ISI-free region.

\section{Acknowledgements}

The author would like to thank the Editor and anonymous reviewers for their helpful comments and suggestions in improving the quality of this 
paper. This work is supported in part by the grants NSC 99-2221-E-006-101 and NSC 100-2221-E-006-172, Taiwan.

\section{Competing interests}

The authors declare that they have no competing interests.

Received: 26 October 2010 Accepted: 19 August 2011

Published: 19 August 2011

\section{References}

1. H Steendam, M Moeneclaey, Analysis and optimization of the performance of OFDM on frequency-selective time-selective fading channels. IEEE Trans Commun. 47(12), 1811-1819 (1999). doi:10.1109/26.809701

2. Y Mostofi, DC Cox, Mathematical analysis of the impact of timing synchronization errors on the performance of an OFDM system. IEEE Trans Commun. 54(2), 226-230 (2006)

3. PH Moose, A technique for orthogonal frequency division multiplexing frequency offset correction. IEEE Trans Commun. 42(10), 2908-2914 (1994). doi:10.1109/26.328961

4. B Stantchev, G Fettweis, Time-variant distortions in OFDM. IEEE Trans Commun. 4(9), 312-314 (2000)

5. JJ van de Beek, M Sandell, PO Borjesson, ML estimation of time and frequency offset in OFDM systems. IEEE Trans Signal Process. 45(7), 1800-1805 (1997). doi:10.1109/78.599949

6. H Minn, VK Bhargava, KB Letaief, A robust timing and frequency synchronization for OFDM systems. IEEE Trans Wirel Commun. 2(4), 822-839 (2003)

7. M Speth, S Fechtel, G Fock, H Meyer, Optimum receiver design for OFDMbased broadband transmission-part Il: a case study. IEEE Trans Commun. 49(4), 571-578 (2001). doi:10.1109/26.917759

8. YH Kim, I Song, S Yoon, SR Park, An efficient frequency offset estimator for OFDM systems and its performance characteristics. IEEE Trans Veh Technol. 50(5), 1307-1312 (2001). doi:10.1109/25.950332

9. G Ren, $\mathrm{Y}$ Chang, $\mathrm{H}$ Zhang, $\mathrm{H}$ Zhang, An efficient frequency offset estimation method with a large range for wireless OFDM systems. IEEE Trans Veh Technol. 56(4), 1892-1895 (2007)

10. M Morelli, C-C Jay Kuo, M-O Pan, Synchronization techniques for orthogonal frequency division multiple access (OFDMA): a tutorial review. IEEE Proc. 95(7), 1394-1427 (2007)

11. TM Schmidl, DC Cox, Robust frequency and timing synchronization for OFDM. IEEE Trans Commun. 45(12), 1613-1621 (1997). doi:10.1109/ 26.650240

12. D Lee, K Cheun, Coarse symbol synchronization algorithms for OFDM systems in multipath channels. IEEE Commun Lett. 6(10), 446-448 (2002). doi:10.1109/LCOMM.2002.804247

13. K Ramasubramanian, K Baum, An OFDM timing recovery scheme with inherent delay-spread estimation, in Proceedings of IEEE GLOBE-COM'01 5 3111-3115 (Nov. 2001)

14. R Negi, JM Cioffi, Blind OFDM symbol synchronization in ISI channels. IEEE Trans Commun. 50(9), 1525-1534 (2002). doi:10.1109/TCOMM.2002.802568

15. C Williams, MA Beach, S McLaughlin, Robust OFDM timing synchronization, in Proceedings of IEEE Vehicular Technology Conference (May 2006)

16. V Krishnamurthy, C Athaudage, D Huang, Adaptive OFDM synchronization algorithms based on discrete stochastic approximation, IEEE Trans Signal Process. 53(4), 1561-1574 (2005)

17. WL Chin, ML Estimation of timing and frequency offsets using distinctive correlation characteristics of OFDM signals over dispersive fading channels. IEEE Trans Veh Technol (Dec. 2010)

18. WL Chin, SG Chen, A blind synchronizer for OFDM systems based on SINR maximization in multipath fading channels. IEEE Trans Veh Technol. 58(2), 625-635 (2009)

19. WL Chin, SG Chen, A low-complexity minimum-interference symbol time estimation for OFDM systems. IEICE Trans Commun. E92-B(5), 1828-1833 (2009). doi:10.1587/transcom.E92.B.1828

20. T Cui, C Tellambura, Power delay profile and noise variance estimation for OFDM. IEEE Commun Lett. 10(1), $25-27$ (2006). doi:10.1109/ LCOMM.2006.1576558

21. Recommendation ITU-R M.1225, in Guideline for Evaluation of Radio Transmission Technologies for IMT-2000 (1997) doi:10.1186/1687-1499-2011-68

Cite this article as: Chin: Channel length assisted symbol

synchronization for OFDM systems in multipath fading channels.

EURASIP Journal on Wireless Communications and Networking 2011 2011:68.

\section{Submit your manuscript to a SpringerOpen ${ }^{\circ}$ journal and benefit from:}

- Convenient online submission

- Rigorous peer review

- Immediate publication on acceptance

- Open access: articles freely available online

- High visibility within the field

- Retaining the copyright to your article

Submit your next manuscript at $\gg$ springeropen.com 